\title{
Further comments on the representation problem for stationary processes ${ }^{\text {th }}$
}

\author{
Stéphane Laurent \\ Université catholique de Louvain, Louvain-la-Neuve, Belgium.
}

\begin{abstract}
We comment on some points about the coding of stochastic processes by sequences of independent random variables. The most interesting question has to do with the standardness property of the filtration generated by the process, in the framework of Vershik's theory of filtrations. Non-standardness indicates the presence of long memory in a purely probabilistic sense. We aim to provide a short, non-technical presentation of Vershik's theory of filtrations.
\end{abstract}

Key words: Standard filtrations, long memory processes

2000 MSC: 60G07, 60G10, 60G25, 37A35, 68P30, 60F20

\section{Introduction}

Rosenblatt (2009) made a comment on a representation problem for stationary processes raised by Lévy (1954), Kallianpur and Wiener (1956) ${ }^{1}$, and Wiener (1958), called the "Lévy-Bernstein-Rosenblatt problem" by Vershik (1995), and remarkably investigated by Rosenblatt $(1959,1960,1963)$, who was the first to spot an interesting mistake in the paper by Kallianpur and Wiener (1956). This problem can be further commented in light of the theory of filtrations in discrete, negative time, which was initiated by Vershik in the early seventies, but entered into the western probabilistic culture only some fifteen years ago.

Three representation problems of stochastic processes in terms of a certain coding of independent random variables are presented in this paper. The first one is the one commented by Rosenblatt (2009). The second one is obtained by dropping time-homogeneity in the first one. The third one is obtained from the second one by further requiring the coding to respect the predictions on the future of the process; this representation is equivalent to standardness of the filtration generated by the process, a notion invented by Vershik (1970, 1973, 1994). As an application of Vershik's theory of filtrations, we shall solve the second problem with the help of Vershik's theorem on lacunary isomorphism.

Our main goal is to provide a short and accessible introduction to Vershik's theory of filtrations. After discussing the three representation problems, we will present the known characterizations of standardness

\footnotetext{
${ }^{2}$ Research supported by IAP research network grant nr. P6/03 of the Belgian government (Belgian Science Policy).

Email address: stephane.laurent@uclouvain.be (Stéphane Laurent)

${ }^{1}$ This paper is unpublished, but Masani (1966) provides a discussion on this work.
} 
of a filtration: the I-cosiness criterion, expressed in terms of "successful couplings" of the filtration, and Vershik's standardness criterion, expressed in terms of asymptotic degeneracy of Vershik's progressive predictions of the filtration, obtained by backward iterating the conditioning of conditional laws given the past.

\section{First representation problem: the "time-homogeneous" coding}

Given a stationary process $\left(X_{n}\right)_{n \in \mathbb{Z}}$, the question is, whether or not there exist a sequence $\left(V_{n}^{\prime}\right)_{n \in \mathbb{Z}}$ of i.i.d. random variables and a measurable function $f$ such that the process $\left(X_{n}^{\prime}\right)_{n \in \mathbb{Z}}$ defined by

$$
X_{n}^{\prime}=f\left(\ldots, V_{n-1}^{\prime}, V_{n}^{\prime}\right)
$$

is a copy of $\left(X_{n}\right)_{n \in \mathbb{Z}}$ ) (i.e., has the same law as $\left(X_{n}\right)_{n \in \mathbb{Z}}$ ). We can interpret the representation (1), as well as the next representations (2) and (3), as a "coding", by considering $\left(X_{n}^{\prime}\right)_{n \in \mathbb{Z}}$ as the "observed" process and $\left(V_{n}^{\prime}\right)_{n \in \mathbb{Z}}$ as the "source" process.

Terming the increasing sequence $\mathcal{F}=\left(\mathcal{F}_{n}\right)_{n \in \mathbb{Z}}$ of $\sigma$ - fields defined by $\mathcal{F}_{n}=\sigma\left(X_{m} ; m \leqslant n\right)$ as the filtration generated by $\left(X_{n}\right)_{n \in \mathbb{Z}}$, a necessary condition for the representation (1) is the tail-triviality of this filtration:

$$
\left(A \in \mathcal{F}_{-\infty}:=\bigcap_{n} \mathcal{F}_{n}\right) \quad \Longrightarrow \quad(\mathbb{P}(A)=0 \text { or } \mathbb{P}(A)=1) .
$$

Indeed, the representation (1) implies that, up to isomorphism, $\mathcal{F}$ is included in the filtration generated by $\left(V_{n}^{\prime}\right)_{n \in \mathbb{Z}}$, and the latter is tail-trivial by Kolmogorov's 0-1 law. We term as Kolmogorovian such a filtration. In the language of ergodic theory, this is expressed by saying that the shift operator acting on the probability space induced by $\left(X_{n}\right)_{n \in \mathbb{Z}}$ is a $K$-automorphism. Hence, as noticed by Rosenblatt (2009), an example of a stationary process generating a Kolmogorovian filtration but for which no representation (1) is possible, is provided by Ornstein's theory developed in the early seventies. The sketch of the argument runs as follows in the language of ergodic theory. The representation (1) implies that the shift induced by $\left(X_{n}\right)_{n \in \mathbb{Z}}$ is a factor of the shift induced by $\left(V_{n}^{\prime}\right)_{n \in \mathbb{Z}}$. The latter is a Bernoulli shift since the $V_{n}^{\prime}$ are independent, and hence each of its factors is also (isomorphic to) a Bernoulli shift thanks to Ornstein's theorem (1970). Thus the existence of a stationary process generating a Kolmogorovian filtration but for which no representation (1) is possible, comes down to the existence of non-Bernoulli K-automorphisms, first established by Ornstein (1973), and the first example of a "natural" non-Bernoulli K-automorphism was given by Kalikow (1982). Kalikow's example is described by Rosenblatt (2009).

\section{Two other representation problems: the "general" and the "real-time" codings}

More interestingly, Feldman and Rudolph (1998) provide an example of a stationary process $\left(X_{n}\right)_{n \in \mathbb{Z}}$ for which there is no way of having a representation (1), but however it is possible to find a copy $\left(X_{n}^{\prime}\right)_{n \in \mathbb{Z}}$ of $\left(X_{n}\right)_{n \in \mathbb{Z}}$ written in the form

$$
X_{n}^{\prime}=f_{n}\left(\ldots, V_{n-1}^{\prime}, V_{n}^{\prime}\right)
$$


where $\left(V_{n}^{\prime}\right)_{n \in \mathbb{Z}}$ is a sequence of i.i.d. random variables and the $f_{n}$ are measurable functions. Actually, Feldman and Rudolph's example is a stationary process generating a standard filtration, or, in other words, it satisfies the stronger representation problem (3), to be introduced below. In fact we shall see in the next paragraph that a representation (2) is always possible for any (possibly nonstationary) process $\left(X_{n}\right)_{n \in \mathbb{Z}}$ generating a Kolmogorovian filtration. This is the only original result of the present paper; our proof will make use of Vershik's theorem on lacunary isomorphism for filtrations. However, this answer to the representation problem (2) is not our motivation for introducing Vershik's theory of filtrations. This theory solves the question of a much more interesting representation problem than (2). Roughly speaking, the representation problem (2) only requires that it is possible to construct a copy $\left(X_{n}^{\prime}\right)_{n \in \mathbb{Z}}$ of $\left(X_{n}\right)_{n \in \mathbb{Z}}$ from $\left(V_{n}^{\prime}\right)_{n \in \mathbb{Z}}$ in a general way, whereas the following question of "real-time" coding is a more interesting one: When is it possible to construct $\left(X_{n}^{\prime}\right)_{n \in \mathbb{Z}}$ from $\left(V_{n}^{\prime}\right)_{n \in \mathbb{Z}}$ in such a way that at each time $n$, the information carried by the past process $\left(V_{k}^{\prime}\right)_{k<n}$ does not disturb the predictions on the future of $\left(X_{n}^{\prime}\right)_{n \in \mathbb{Z}}$ ? More rigorously, this representation problem is:

$$
\left\{\begin{array}{l}
X_{n}^{\prime}=f_{n}\left(\ldots, V_{n-1}^{\prime}, V_{n}^{\prime}\right) \\
\mathbb{P}\left(X_{n}^{\prime} \in \cdot \mid V_{k}^{\prime} ; k<n\right)=\mathbb{P}\left(X_{n}^{\prime} \in \cdot \mid X_{k}^{\prime} ; k<n\right)
\end{array}\right.
$$

With the terminology of the next paragraph, the question of the representation (3) for the (possibly nonstationary) process $\left(X_{n}\right)_{n \in \mathbb{Z}}$ is exactly the question whether the filtration generated by $\left(X_{n}\right)_{n \in \mathbb{Z}}$ is standard, a notion to be explained below, lying at the heart of Vershik's theory of filtrations. The second condition in (3), expressed in the next paragraph in terms of the immersion property for filtrations, is quite natural and is often "hidden" in the probabilist literature. For example, if $\left(X_{n}^{\prime}\right)_{n \in \mathbb{Z}}$ is a Markov process, then this condition is another way to say that $\left(X_{n}^{\prime}\right)_{n \in \mathbb{Z}}$ is still Markovian with respect to the (possibly larger) filtration generated by $\left(V_{n}^{\prime}\right)_{n \in \mathbb{Z}}$. We notice that this condition is fulfilled in all Rosenblatt's examples of representations (1) for stationary processes. Thus, the works of Rosenblatt provide some examples of standard filtrations.

\section{Standard and non-standard filtrations}

Filtrations are the relevant mathematical objects to formulate the representations (2) and (3). Indeed, denoting by $\mathcal{F}=\left(\mathcal{F}_{n}\right)_{n \in \mathbb{Z}}$ the filtration generated by $\left(X_{n}\right)_{n \in \mathbb{Z}}$, the representation (2) exactly says that, up to isomorphism, $\mathcal{F}$ is included in the filtration $\mathcal{G}^{\prime}$ generated by the sequence $\left(V_{n}^{\prime}\right)_{n \in \mathbb{Z}}$ of independent random variables, whereas the stronger representation (3) says that, up to isomorphism, $\mathcal{F}$ is immersed in $\mathcal{G}^{\prime}$, according to the following definition.

Definition. A filtration $\mathcal{F}$ is immersed in a filtration $\mathcal{G}$ if $\mathcal{F}$ is included in $\mathcal{G}$ and, for each time $n$, the $\sigma$ - field $\mathcal{F}_{n+1}$ is conditionally independent of $\mathcal{G}_{n}$ given $\mathcal{F}_{n}$. 
Definitions. A filtration $\mathcal{F}$ is standard if, up to isomorphism, it is immersed in a standard non-atomic filtration, that is, a filtration generated by a sequence of i.i.d. random variables uniformly distributed on the interval $[0,1]$.

Proposition. A filtration $\mathcal{F}$ is standard if and only if, up to isomorphism, it is immersed in a filtration generated by a sequence of independent random variables.

We refer to Émery and Schachermayer (2001) or Laurent (2010a) for a proof of the previous proposition, which stems from elementary considerations. It shows that the laws of the independent random variables $V_{n}^{\prime}$ play no role in the representation (3).

Thus, the representation problem (3) for the (possibly nonstationary) process $\left(X_{n}\right)_{n \in \mathbb{Z}}$ is exactly the question of standardness of the filtration it generates. Vershik $(1970,1973,1994)$ has stated (among other things) a necessary and sufficient criterion for standardness and has exhibited non trivial examples of standard and non-standard filtrations. His works are written in Rokhlin's language (Rokhlin, 1952) and their rephrasement in the language of stochastic processes such as the one used in the present paper, more familiar to probabilists, has been initiated by Émery and Schachermayer (2001). Laurent (2004, 2010a, 2010b) gives simpler approaches for proving some of the known results and further investigates Vershik's theory in the language of stochastic processes. We will present the known characterizations of standardness in the next paragraph.

Note that, obviously, each $\sigma$ - field $\mathcal{F}_{n}$ of a standard filtration $\mathcal{F}$ is essentially separable, that is, countably generated modulo null events, because this property is inherited by inclusion. The essential separability of the $\mathcal{F}_{n}$ is the usual standing assumption in the study of filtrations, since it is always fulfilled for filtrations generated by a sequence of random variables taking their values in separable metric spaces. Under this standing assumption, standardness is an asymptotic property when $n \rightarrow-\infty$ : standardness of a filtration $\mathcal{F}=\left(\mathcal{F}_{n}\right)_{n \in \mathbb{Z}}$ with time-axis $\mathbb{Z}$ is equivalent to standardness of its restriction $\mathcal{F}=\left(\mathcal{F}_{n}\right)_{n \leqslant 0}$ to the time-axis $-\mathbb{N}$.

Non-standardness of a Kolmogorovian filtration indicates the presence of a long memory in spite of the tail-triviality. Bressaud \& al (2006) give a sufficient condition for a stationary process $\left(X_{n}\right)_{n \in \mathbb{Z}}$ taking only two possible values to generate a standard filtration $\mathcal{F}$. Their condition is expressed in terms of the dependence on the past $\left(X_{n-1}, X_{n-2}, \ldots\right)$ of the conditional probability $\mathbb{P}\left(X_{n} \in \cdot \mid X_{n-1}, X_{n-2}, \ldots\right)$, under the standing assumption that this conditional probability almost surely takes positive values; it is satisfied in particular when $\left(X_{n}\right)_{n \in \mathbb{Z}}$ is a stationary finite-order Markov chain. Some improvements of Bressaud \& al's result and generalizations to the case of a finite state space have been obtained by Ceillier (2009).

Long-memory phenomena are of interest in the theory of stationary time series which are most often 
Gaussian processes. In the case when $\mathcal{F}$ is the filtration generated by a stationary Gaussian process $\left(X_{n}\right)_{n \in \mathbb{Z}}$, Blum \& Hanson (1962) provide a theorem from which we can deduce that standardness of $\mathcal{F}$ holds whenever $\mathcal{F}$ is Kolmogorovian, and they give a necessary and sufficient condition for $\mathcal{F}$ to be Kolmogorovian in terms of the asymptotic behavior of the sequence $\left(\rho_{n}\right)$ of covariances defined by $\rho_{i-j}=\operatorname{Cov}\left(X_{i}, X_{j}\right)=\operatorname{Cov}\left(X_{0}, X_{i-j}\right)$. Actually, as shown by Leuridan (2009) by a straightforward adaptation of the mathematics used by Blum \& Hanson (1962), it is also true that every Kolmogorovian filtration generated by a Gaussian process is standard, without supposing stationarity.

Of particular interest is the following theorem established by Vershik (1968), with the help of which we shall prove, as announced in the previous paragraph, that the representation problem (2) has a positive answer for any process $\left(X_{n}\right)_{n \in \mathbb{Z}}$ generating a Kolmogorovian filtration.

VERSHIK'S THEOREM ON LACUNARY ISOMORPHISM. Any Kolmogorovian filtration $\mathcal{F}$ is lacunary isomorphic to a standard filtration, that is, there exists a standard filtration extracted from $\mathcal{F}$. (The precise meaning will soon be made more clear.)

COROLlary. Up to isomorphism, any Kolmogorovian filtration $\mathcal{F}$ is included in a standard non-atomic filtration.

Proof. According to Vershik's theorem on lacunary isomorphism, there exists a strictly increasing sequence $\phi: \mathbb{Z} \rightarrow \mathbb{Z}$ such that the "extracted" filtration $\left(\mathcal{F}_{\phi(n)}\right)_{n \in \mathbb{Z}}$ is standard. In particular this filtration is included, up to isomorphism, in the filtration generated by a sequence $\left(U_{n}\right)_{n \in \mathbb{Z}}$ of i.i.d. random variables, each $U_{n}$ being uniformly distributed on $[0,1]$. By enriching the probability space if needed, we may assume that there is another such sequence $\left(U_{n}^{\prime}\right)_{n \in \mathbb{Z}}$ of random variables that is independent of $\left(U_{n}\right)_{n \in \mathbb{Z}}$. We define $U_{k}^{*}=U_{n+1}$ if $k=\phi(n)+1$ for some $n$ and $U_{k}^{*}=U_{k}^{\prime}$ for all other values of $k$. Obviously, $\mathcal{F}$ is included in the standard non-atomic filtration generated by $\left(U_{n}^{*}\right)_{n \in \mathbb{Z}}$.

\section{Characterizations of standardness}

Below we list the known criteria for standardness, without rigorously defining them. We refer to Émery (2002) for a noteworthy survey on Vershik's theory of filtrations, as well as original examples. Recall that we make the standing assumption that each $\sigma$ - field $\mathcal{F}_{n}$ is essentially separable. As standardness is an asymptotic property when $n \rightarrow-\infty$, it is not restrictive, and more convenient, to deal with filtrations $\mathcal{F}=\left(\mathcal{F}_{n}\right)_{n \leqslant 0}$ indexed by the time-axis $-\mathbb{N}$. Hereafter, we shall always make this standing assumption.

1. Vershik's standardness criterion. Given a random variable $X$ measurable with respect to the final 
$\sigma$ - field $\mathcal{F}_{0}$, all our knowledge about $X$ at time -1 is contained in the conditional law $\mathcal{L}\left(X \mid \mathcal{F}_{-1}\right)$. But it is a serious mistake to think that the conditional law $\mathcal{L}\left(X \mid \mathcal{F}_{-2}\right)$ contains all our knowledge about $X$ at time -2 , which is actually contained in the conditional law of the conditional law $\mathcal{L}\left(\mathcal{L}\left(X \mid \mathcal{F}_{-1}\right) \mid \mathcal{F}_{-2}\right)$. We recursively define the sequence of Vershik's progressive predictions $\left(\pi_{n} X\right)_{n \leqslant 0}$ of $X$ by iterating this procedure: we put $\pi_{0} X=X$ and $\pi_{n-1} X=\mathcal{L}\left(\pi_{n} X \mid \mathcal{F}_{n-1}\right)$. Vershik's standardness criterion says that $\mathcal{F}$ is standard if and only if for any $\mathcal{F}_{0}$-measurable random variable $X$, the $n$-th progressive prediction $\pi_{n} X$ of $X$ becomes arbitrarily close to a constant with arbitrarily high probability as $n$ goes small. This is a useful criterion for proving theoretical results. Laurent (2010b) gives a short proof of Vershik's theorem on lacunary isomorphism based on Vershik's standardness criterion. In order to rigorously formulate Vershik's standardness criterion, the state spaces of Vershik's progressive predictions $\pi_{n} X$ are endowed with Kantorovich metrics, well-known in the study on the sometimes called "Monge-Kantorovich transportation problem". An excellent reference on the Kantorovich metric is the paper by Vershik (2006).

2. I-cosiness criterion. Due to the link between the Kantorovich metric and joinings (or couplings) of random variables, Vershik's standardness criterion is characterized by the I-cosiness criterion in terms of joinings of filtrations. This criterion, introduced by Émery and Schachermayer (2001), is mainly inspired from the notion of cosiness devised by Tsirelson (1997) in the framework of continuous time. To a statistician, it could remind the "coupling from the past" method. It says that a filtration is standard if and only if for every random variable $X$ measurable with respect to the final $\sigma$-field $\mathcal{F}_{0}$, there exists a joining $\left(\mathcal{F}^{\prime}, \mathcal{F}^{\prime \prime}\right)$ of the filtration $\mathcal{F}$ (that is, a pair of two filtrations $\mathcal{F}^{\prime}$ and $\mathcal{F}^{\prime \prime}$ each isomorphic to $\mathcal{F}$ and both immersed in a same bigger filtration), independent in small time (that is, $\mathcal{F}_{n_{0}}^{\prime}$ and $\mathcal{F}_{n_{0}}^{\prime \prime}$ are independent for some time $n_{0} \leqslant 0$ ), and for which the respective copies $X^{\prime}$ and $X^{\prime \prime}$ of $X$ are arbitrarily close with high probability. For example, Laurent (2010a) shows how to deduce from well-known results on couplings of Markov chains that a stationary Markov chain on a denumerable state space generates an I-cosy filtration whenever this filtration is Kolmogorovian (which is equivalent for the Markov chain to be irreducible, recurrent and aperiodic). The standardness of such filtrations was originally established by Rosenblatt (1960) by others considerations.

3. Combinatorial Vershik's standardness criterion. In his pioneering works, Vershik focused on $\left(r_{n}\right)$-adic filtrations, that is, filtrations $\mathcal{F}$ for which, for each $n \leqslant 0$, there exists a random variable $V_{n}$ uniformly distributed on a finite set with $r_{n}$ elements, independent of $\mathcal{F}_{n-1}$ and such that $\mathcal{F}_{n}=\mathcal{F}_{n-1} \vee \sigma\left(V_{n}\right)$. For such filtrations, Vershik has stated a combinatorial form of his standardness criterion, involving tree automorphisms. In the language of stochastic processes, this criterion is close to Vershik's self-joining criterion introduced by Laurent (2010a), which is a particular case of the I-cosiness criterion when the joinings $\left(\mathcal{F}^{\prime}, \mathcal{F}^{\prime \prime}\right)$ are given by some underlying tree automorphisms; Laurent (2010a) elementarily 
shows that the I-cosiness criterion reduces to Vershik's self-joining criterion for $\left(r_{n}\right)$-adic filtrations with the help of Birkhoff-Von Neumann's theorem (permutation matrices are extreme doubly stochastic matrices).

In fact, originally, Vershik's theory was mainly oriented towards the characterization of $\left(r_{n}\right)$-adic filtrations generated by a sequence of independent random variables. Actually standardness provides such a characterization, as testified by the following important theorem due to Vershik.

Theorem. Let $\mathcal{F}=\left(\mathcal{F}_{n}\right)_{n \leqslant 0}$ be a filtration such that $\mathcal{F}_{n}=\mathcal{F}_{n-1} \vee \sigma\left(V_{n}\right)$ for each $n \leqslant 0$ where $V_{n}$ is a random variable independent of $\mathcal{F}_{n-1}$, having either an uniform distribution on a finite set or a diffuse law (a continuous distribution). Then $\mathcal{F}$ is standard if and only if $\mathcal{F}$ is generated by a sequence of independent random variables.

\section{A stationary process whose filtration is non-standard: Vershik's Example 3}

Vershik (1995) provides the following example of a stationary process whose filtration is Kolmogorovian but non-standard.

Let $A$ be a finite set and $\mu$ the uniform probability measure on $A$. Vershik's example is a stationary Markov process $\left(V_{n}\right)_{n \in \mathbb{Z}}$. Its state space is the set $A^{\mathbb{N}}$ made of all infinite words $v=v^{0} v^{1} v^{2} \ldots$ and endowed with the product measure $\mu^{\mathbb{N}}$ (the letters are independent and uniform). The Markov transition is

$$
v^{0} v^{1} v^{2} v^{3} v^{4} \ldots \longmapsto \begin{cases}v^{0} v^{2} v^{4} \ldots & \text { with probability } 1 / 2, \\ v^{1} v^{3} v^{5} \ldots & \text { with probability } 1 / 2 .\end{cases}
$$

In other words, $V_{n}$ is uniformly chosen to be either the "even half" or the "odd half" of $V_{n-1}$.

Vershik showed that the process $\left(V_{n}\right)_{n \leqslant 0}$ generates a Kolmogorovian but non-standard filtration $\mathcal{F}$ with the help of his standardness criterion. We note in passing that we do not know whether $\left(V_{n}\right)_{n \leqslant 0}$ satisfies the representation (1).

Smorodinsky (1998) provides a proof that $\mathcal{F}$ is not standard in the language of stochastic processes. Smorodinsky's proof involves the I-cosiness criterion, but without naming it; a copied proof using the language of I-cosiness is provided by Émery \& Schachermayer (2001).

\section{From Vershik's theory to Brownian filtrations}

Vershik's theory on filtrations in discrete, negative time, remained unknown to the western probabilistic culture for about twenty-five years, until Dubins, Feldman, Smorodinsky and Tsirelson (1996) used Vershik's standardness criterion to show that the (continuous-time) filtration generated by a Brownian motion does not necessarily remain generated by a Brownian motion after an equivalent change of probability has been 
performed. We refer to Émery (2000) for a noteworthy survey on Vershik's theory and its relations with some questions on Brownian motion.

Émery (2005) shows that the continuous-time analogue of the I-cosiness criterion characterizes Brownian filtrations among filtrations which are "Brownian after zero"; with the help of this criterion, Émery (2009) gives the first example of a filtration which is Brownian, but for which no generating Brownian motion has constructively been exhibited.

\section{Acknowledgements}

I am indebted to the IAP research network for financial support, and to the referre for his/her careful reading of the paper which helped to improve the presentation.

\section{References}

[1] J. Blum, D. Hanson, 1962. On a Problem in Hilbert Space with Applications. Journal of Mathematics and Mechanics 11:3, 497-501.

[2] X. Bressaud, A. Maass, S. Martinez, J. San Martin, 2006. Stationary processes whose filtrations are standard. Annals of Probability 34:4, 1589-1600.

[3] G. Ceillier, 2009. PhD dissertation, in preparation. Institut Fourier de Grenoble.

[4] L.E. Dubins, J. Feldman, M. Smorodinsky, B. Tsirelson, 1996. Decreasing sequences of $\sigma$-fields and a measure change for Brownian motion. Annals of Probability 24:2, 882-904.

[5] M. Émery, 2000. De la théorie de Vershik au mouvement brownien, via des idées de Tsirelson. Séminaire Bourbaki 43, 2000/2001, Exp. No. 882, 63-83.

[6] M. Émery, 2002. Old and new tools in the theory of filtrations. In: Dynamics and Randomness, ed by A. Maass, S. Martinez, J. San Martin. Kluwer Academic Publishers, Massachusetts, pp 125-146.

[7] M. Émery, 2005. On certain almost Brownian filtrations. Annales de l'institut Henri Poincaré (B) Probabilités et Statistiques, 41:3, 285-305

[8] M. Émery, 2009. Recognising whether a filtration is Brownian: a case study. Séminaire de probabilités XLII, Springer Lectures Notes in Math. 1979, 383-396.

[9] M. Émery, W. Schachermayer, 2001. On Vershik's standardness criterion and Tsirelson's notion of cosiness. Séminaire de probabilités XXXV, Springer Lectures Notes in Math. 1755, 265-305.

[10] J. Feldman, D. Rudolph, 1998. Standardness of sequences of $\sigma$-fields given by certain endomorphisms. Fundamenta Mathematicae 157, 175-189.

[11] S.A. Kalikow, 1982. T, $T^{-1}$ transformation is not loosely Bernoulli. Ann. Math. 115, 393-409.

[12] G. Kallianpur, N. Wiener, 1956 (unpublished manuscript). Non-linear prediction. Technical Report 1, Office of Naval Research

[13] S. Laurent, 2004. Filtrations à temps discret négatif. PhD dissertation, IRMA, Université de Strasbourg.

[14] S. Laurent, 2010a. On standardness and I-cosiness. To appear in: Séminaire de Probabilités XLIII. 
[15] S. Laurent, 2010b. On Vershikian and I-cosy random variables and filtrations. To appear in: Teoriya Veroyatnostei i ee Primeneniya.

[16] C. Leuridan, 2009. Filtration d'une suite de variables aléatoires gaussiennes. Privately circulated manuscript.

[17] P. Lévy, 1954. Théorie de l'addition des variables aléatoires, 2nd ed. Gauthier Villars, Paris.

[18] P. Masani, 1966. Wiener's contribution to generalized harmonic analysis. Bull. AMS 72, $73-125$.

[19] D. S. Ornstein, 1970. Factors of Bernoulli shifts are Bernoulli shifts. Advances in Math. 5, 349-364.

[20] D. S. Ornstein, 1973. An example of a Kolmogorov automorphism that is not a Bernoulli shift. Adv. in Math. 10 , 49-62.

[21] V. A. Rokhlin, 1952. On the fundamental ideas of measure theory. Amer. Math. Soc. Translation 71, 1-53.

[22] M. Rosenblatt, 1959. Stationary processes as shifts of functions of independent random variables. Journal of Mathematics and Mechanics 8:5, 665-681.

[23] M. Rosenblatt, 1960. Stationary Markov Chains and Independent Random Variables. Journal of Mathematics and Mechanics 9:6, 945-949.

[24] M. Rosenblatt, 1963. The Representation of a Class of Two State Stationary Processes in Terms of Independent Random Variables. Journal of Mathematics and Mechanics 12:5, 721-730.

[25] M. Rosenblatt, 2009. A comment on a conjecture of N. Wiener. Statistics and Probability Letters 79, 347-348.

[26] M. Smorodinsky, 1998. Processes with no standard extension. Israel Journal of Mathematics $107,327-331$.

[27] B. Tsirelson, 1997. Triple points: from non-Brownian filtrations to harmonic measures. Geometric And Functional Analysis 7, 1096-1142.

[28] A.M. Vershik, 1968. Theorem on lacunary isomorphisms of monotonic sequences of partitions. Funktsional'nyi Analiz i Ego Prilozheniya 2:3, 17-21. English translation, 1968: Functional Analysis and Its Applications 2:3, $200-203$.

[29] A. M. Vershik, 1970. Decreasing sequences of measurable partitions, and their applications. Dokl. Akad. Nauk SSSR 193, 748-751. English translation, 1970: Soviet mathematics Doklady 11, 1007-1011.

[30] A. M. Vershik, 1973. Approximation in measure theory (In Russian). PhD dissertation, Leningrad Univ. (expanded and updated version: [31])

[31] A. M. Vershik, 1994. The theory of decreasing sequences of measurable partitions (in Russian). Algebra i Analiz 6:4, 1-68. English translation, 1995: St. Petersburg Mathematical Journal 6:4, 705-761.

[32] A.M. Vershik, 2004. The Kantorovich metric: the initial history and little-known applications (in Russian). Representation theory, dynamical systems. Part XI, Special issue, Zap. Nauchn. Sem. POMI 312, POMI, St. Petersburg, 69-85. English translation, 2006: J. Math. Sci. (New York) 133:4, 1410-1417.

[33] N. Wiener, 1958. Nonlinear Problems in Random Theory. MIT Press, Cambridge, MA. 